OPEN ACCESS

Edited by:

Loredana Pagano,

University of Turin, Italy

Reviewed by:

Erivelto Martinho Volpi,

Centro de referencia no ensino do diagnóstico por imagem (CETRUS),

Brazil

Leonardo Rossi,

University of Pisa, Italy

${ }^{*}$ Correspondence:

Yi-Wei Kuo

harry.k101@msa.hinet.net

Che-Wei Wu

cwwu@kmu.edu.tw

${ }^{\dagger}$ These authors have contributed equally to this work

Specialty section:

This article was submitted to

Thyroid Endocrinology,

a section of the journal

Frontiers in Endocrinology

Received: 18 November 2021

Accepted: 11 January 2022

Published: 10 February 2022

Citation:

Lu I-C, Hsu C-D, Chang P-Y,

Wu S-H, Huang T-Y, Lin $Y-C$,

Ko H-Y, Dionigi G, Chai $Y$ J,

Chiang F-Y, Kuo Y-W and Wu C-W

(2022) A Surgeon-Centered

Neuromuscular Block Protocol

Improving Intraoperative

Neuromonitoring Outcome

of Thyroid Surgery.

Front. Endocrinol. 13:817476.

doi: 10.3389/fendo.2022.817476

\section{A Surgeon-Centered Neuromuscular Block Protocol Improving Intraoperative Neuromonitoring Outcome of Thyroid Surgery}

\author{
I-Cheng Lu ${ }^{1,2}$, Chiung-Dan Hsu ${ }^{1}$, Pi-Ying Chang ${ }^{3}$, Sheng-Hua Wu ${ }^{2,3}$, Tzu-Yen Huang ${ }^{2,4}$, \\ Yi-Chu Lin ${ }^{4}$, How-Yun Ko ${ }^{4}$, Gianlorenzo Dionigi ${ }^{5,6}$, Young Jun Chai ${ }^{7}$, Feng-Yu Chiang ${ }^{8}$, \\ Yi-Wei Kuo ${ }^{9 *}$ and Che-Wei Wu ${ }^{2,4^{*}+}$ \\ ${ }^{1}$ Department of Anesthesiology, Kaohsiung Municipal Siaogang Hospital, Kaohsiung Medical University Hospital, Kaohsiung \\ Medical University, Kaohsiung, Taiwan, ${ }^{2}$ Faculty of Medicine, College of Medicine, Kaohsiung Medical University, Kaohsiung, \\ Taiwan, ${ }^{3}$ Department of Anesthesiology, Kaohsiung Municipal Ta-Tung Hospital, Kaohsiung Medical University Hospital, \\ Kaohsiung Medical University, Kaohsiung, Taiwan, ${ }^{4}$ Department of Otorhinolaryngology, Kaohsiung Medical University \\ Hospital, Kaohsiung Medical University, Kaohsiung, Taiwan, ${ }^{5}$ Division of General Surgery, Endocrine Surgery Section, Istituto \\ Auxologico Italiano IRCCS (Istituto di ricovero e cura a carattere scientifico), Milan, Italy, ${ }^{6}$ Department of Pathophysiology and \\ Transplantation, University of Milan, Milan, Italy, ${ }^{7}$ Department of Surgery, Seoul National University College of Medicine, \\ Seoul Metropolitan Government-Seoul National University Boramae Medical Center, Transdisciplinary Department of \\ Medicine \& Advanced Technology, Seoul National University Hospital, Seoul, South Korea, ${ }^{8}$ Department of Otolaryngology, \\ E-Da Hospital, I-Shou University, Kaohsiung, Taiwan, ${ }^{9}$ Department of Anesthesiology, Kaohsiung Medical University \\ Hospital, Kaohsiung Medical University, Kaohsiung, Taiwan
}

Background: Neuromuscular blocking agents provide muscular relaxation for tracheal intubation and surgery. However, the degree of neuromuscular block may disturb neuromuscular transmission and lead to weak electromyography during intraoperative neuromonitoring. This study aimed to investigate a surgeon-friendly neuromuscular block degree titrated sugammadex protocol to maintain both intraoperative neuromonitoring quality and surgical relaxation during thyroid surgery.

Methods: A total of 116 patients were enrolled into two groups and underwent elective thyroid surgery with intraoperative neuromonitoring. All patients followed a standardized intraoperative neuromonitoring protocol with continuous neuromuscular transmission monitoring and received $0.6 \mathrm{mg} / \mathrm{kg}$ rocuronium for tracheal intubation. Patients were allocated into two groups according to the degree of neuromuscular block when the anterior surface of the thyroid gland was exposed. The neuromuscular block degree was assessed by the train-of-four (TOF) count and ratio. Patients in group I received sugammadex $0.25 \mathrm{mg} / \mathrm{kg}$ for non-deep neuromuscular block degree (TOF count = 1 4). Patients in group II were administered sugammadex $0.5 \mathrm{mg} / \mathrm{kg}$ for deep neuromuscular block degree (TOF count $=0$ ). The quality of the intraoperative neuromonitoring was measured using the $V_{1}$ electromyography (EMG) amplitude. An amplitude less than $500 \mu \mathrm{V}$ and greater than $500 \mu \mathrm{V}$ was defined as weak and satisfactory, respectively. 
Results: The quality of the intraoperative neuromonitoring was not different between groups I and || (satisfactory/weak: $75 / 1$ vs. 38/2, $P=0.14$ ). The quality of surgical relaxation was acceptable after sugammadex injection and showed no difference between groups [55/76 (72.3\%) in group I vs. 33/40 (82.5\%) in group II, $P=0.23]$.

Conclusions: This surgeon-centered sugammadex protocol guided by neuromuscular block degree ( $0.5 \mathrm{mg} / \mathrm{kg}$ for deep block and $0.25 \mathrm{mg} / \mathrm{kg}$ for others) showed comparably high intraoperative neuromonitoring quality and adequate surgical relaxation. The results expanded the practicality of sugammadex for precise neuromuscular block management during monitored thyroidectomy.

Keywords: intraoperative neuromonitoring (IONM), thyroid surgery, recurrent laryngeal nerve (RLN), neuromuscular block degree, sugammadex

\section{INTRODUCTION}

Intraoperative neuromonitoring (IONM) has gained increasing popularity during thyroid surgery in recent years. IONM is an adjunct tool for the identification and localization, detecting anatomical variations, differentiating mechanisms of injury, and predicting the postoperative function of the recurrent laryngeal nerve (RLN) (1-12). The goal of IONM is to diminish the occurrence of RLN injury and vocal cord paralysis associated with thyroid and parathyroid surgery.

Both good contact of surface electrodes (13-19) and adequate reversal of neuromuscular blockade (NMB) (20-27) are prerequisites for successful IONM of the RLN. For adequate relaxation to facilitate tracheal intubation and surgery, a profound or deep NMB is desirable. A proper reversal of the $\mathrm{NMB}$ is mandatory for functional IONM via evoked electromyogram (EMG) signals. Recently, sugammadex following rocuronium during anesthesia induction has been reported as an effective NMB regimen for successful IONM in animal and human clinical studies involving thyroid surgery (28-30).

Various sugammadex regimens have been reported. Lowdose sugammadex can enhance spontaneous neuromuscular functional recovery, while high-dose sugammadex can result in undesirable involuntary movement during surgery. In our previous report, sugammadex at a dose of $0.5 \mathrm{mg} / \mathrm{kg}$ induced $\mathrm{NMB}$ reversal from rocuronium $0.6 \mathrm{mg} / \mathrm{kg}$ and provided highquality monitoring of the RLN for thyroid surgery (31). Chai et al. (32) demonstrated that sugammadex at either 1 or $2 \mathrm{mg} / \mathrm{kg}$ resulted in high-quality IONM. The bucking effect of sugammadex is dose-related, such that $2 \mathrm{mg} / \mathrm{kg}$ sugammadex was associated with up to $35 \%$ higher effects than $1 \mathrm{mg} / \mathrm{kg}$ sugammadex (32). However, the titration of sugammadex dosage according to the degree of $\mathrm{NMB}$ has not been investigated.

This study aimed to establish a surgeon-friendly protocol by titrating the sugammadex dose based on the degree of NMB before initial vagus nerve stimulation $\left(\mathrm{V}_{1}\right)$ to maintain both surgical relaxation and IONM quality during thyroid surgery. We hypothesized that sugammadex titrated at a dose of $0.25 \mathrm{mg}$ / $\mathrm{kg}$ is effective for the reversal of rocuronium-induced moderate
$\mathrm{NMB}$ and can provide effective IONM with sufficient surgical relaxation. We compared this protocol with $0.5 \mathrm{mg} / \mathrm{kg}$ sugammadex for deep $\mathrm{NMB}$, which was routinely administered at our institution.

\section{METHODS}

\section{Patient Data}

This retrospective observational study was approved by the institutional review board of Kaohsiung Medical University Hospital [KMUHIRB-E(I)-20210070] and registered at ClinicalTrials.gov (NCT 04982185). Patients who underwent elective total thyroidectomy or total lobectomy with routine IONM were included between August 1, 2019, and July 31, 2020. Patients who met the following exclusion criteria were excluded from the study: age $\leq 20$ years, American Society of Anesthesiologists (ASA) status of $\geq 4$, vocal cord palsy, or previous thyroid surgery. All operations were performed by the same surgeon, and anesthesia was administered by two experienced anesthesiologists. The neuromonitoring setup, surgical procedures, and loss of signal algorithm followed the International Neural Monitoring Study Group Guidelines (1, 5, 11, 12).

\section{Anesthesia}

Upon arrival at the operating room, each patient was placed under standard physiological monitoring (oximetry, electrocardiography, non-invasive blood pressure, and capnography). Before anesthesia induction, a donut pad beneath the neck was placed for thyroidectomy. An oral endotracheal tube with a 7.0- and 7.5- $\mathrm{mm}$ internal diameter was placed for female and male patients, respectively.

The NMB degree was continuously monitored by the trainof-four (TOF) count and ratio derived from the adductor pollicis muscle. Anesthetic depth was assessed using the response entropy (RE) or bispectral index (BIS). Anesthesia induction was initiated with fentanyl $(1 \mu \mathrm{g} / \mathrm{kg})$, lidocaine $(1 \mathrm{mg} / \mathrm{kg})$, and propofol $(1.5-2 \mathrm{mg} / \mathrm{kg})$. When loss of consciousness was identified, rocuronium $(0.6 \mathrm{mg} / \mathrm{kg})$ was administered to induce 
NMB in all patients. When the maximum NMB was achieved, the anesthesiologist performed tracheal intubation using a Trachway video intubating stylet (Biotronic Instrument Enterprise Ltd., Tai Chung, Taiwan). The position of the endotracheal tube was confirmed by end-tidal $\mathrm{CO}_{2}$ and auscultation.

Anesthesia was maintained with sevoflurane and propofol target-controlled infusion with an Orchestra ${ }^{\mathrm{TM}}$ infusion pump (Fresenius Vial, France). The effect-site concentration of propofol was maintained at $1-1.5 \mu \mathrm{g} / \mathrm{ml}$. Two registered nurse anesthetists followed the standardized anesthesia regimen according to our institution's protocol and adjusted the inhaled sevoflurane concentration to maintain an entropy or BIS value between 40 and 60 . A bolus of fentanyl $0.5 \mu \mathrm{g} / \mathrm{kg}$ was administered before the skin incision.

\section{Study Flowchart}

The timing of sugammadex administration was decided by the surgeon after the initial surgical steps of subplatysmal flap creation and strap muscle separation to expose the anterior surface of the thyroid gland. Patients were allocated into two groups according to the degree of $\mathrm{NMB}$ before sugammadex administration. A TOF count of 0 was defined as deep NMB. A TOF count of 1-3 was defined as moderate neuromuscular block and 4 as recovery (33). Patients with non-deep NMB (group I) received sugammadex $0.25 \mathrm{mg} / \mathrm{kg}$. Patients with deep NMB (group II) were administered sugammadex $0.5 \mathrm{mg} /$ kg (Figure 1). All patients followed the standard anesthesia protocol for monitored thyroidectomy (Table 1).

\section{Surgical Techniques and Intraoperative Neuromonitoring Setup}

A lower neck skin incision was made along the skin crease. Subcutaneous fat and platysma were divided, and a subplatysmal dissection was made above the incision up to the level of the thyroid cartilage. The fascia between the strap muscles was divided, and the anterior surface of the thyroid gland was exposed. After resection of the pyramidal lobe, a pair of subdermal electrodes (length, $12.0 \mathrm{~mm}$; diameter, $0.4 \mathrm{~mm}$; Medtronic, Jacksonville, FL) was inserted into the subperichondrium of the thyroid cartilage lamina on both sides (34). The thyroid cartilage electrodes were connected to the nerve integrity monitoring (NIM). The elicited EMG signals with electrode leads were documented as channels 1 and 2 simultaneously. The NIM system generated stimuli with a time window set to $50 \mathrm{~ms}$ and an amplitude scale set to $0.2 \mathrm{mV} /$ division. The pulsed stimuli were 100 $\mu$ s in duration and $4 \mathrm{~Hz}$ in frequency. Event capture was activated at a threshold of $100 \mu \mathrm{V}$. The intraoperative standardized IONM protocol routinely followed the departmental guidelines. The highest EMG amplitudes were recorded. $\mathrm{V}_{1}$ signal represents vagal stimulation before dissection, $\mathrm{R}_{1}$ signal represents $\mathrm{RLN}$ stimulation at first identification, $R_{2}$ signal represents $R L N$ stimulation after complete dissection, and $\mathrm{V}_{2}$ signal represents repeat vagal stimulation after resection of the thyroid.

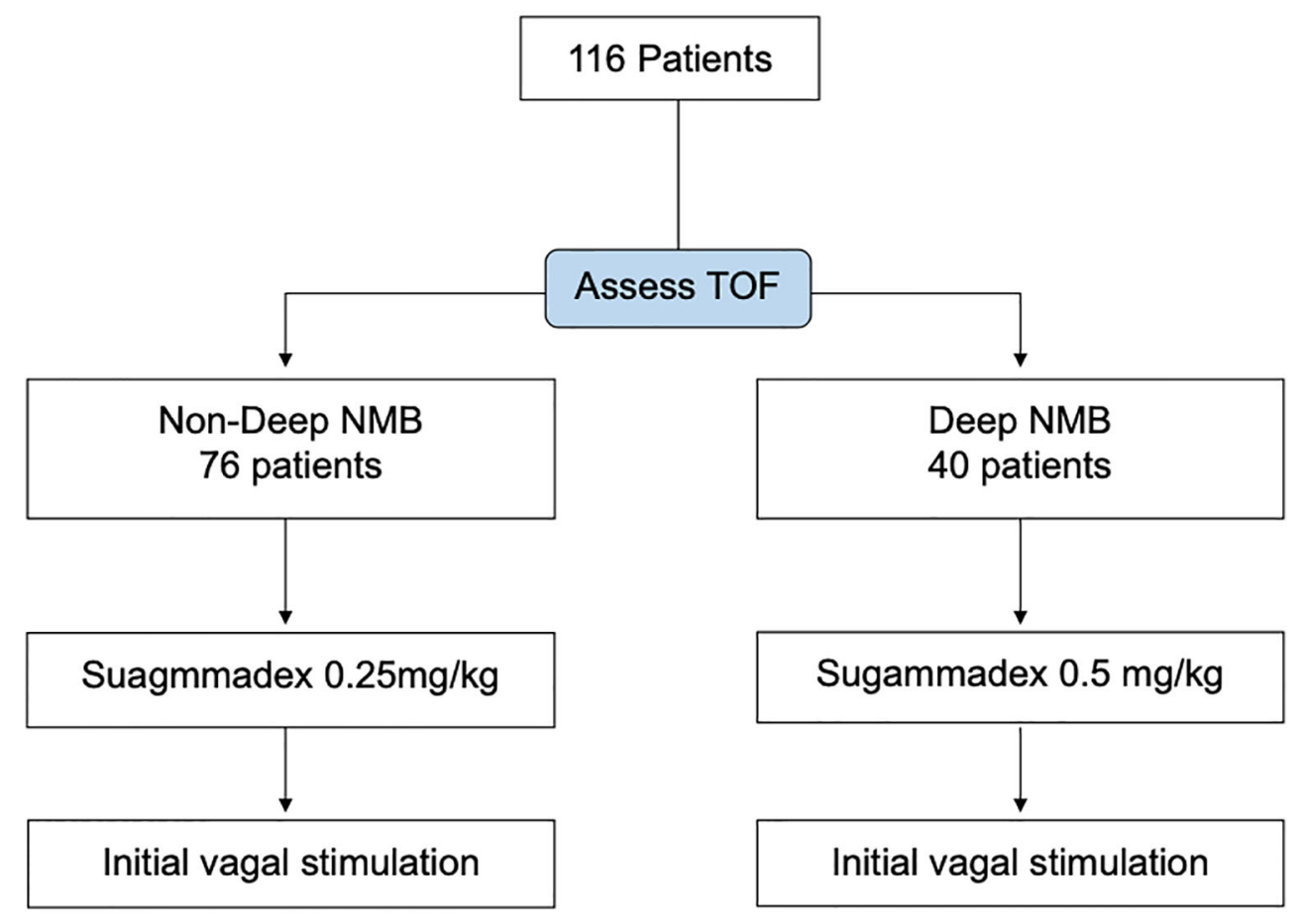

FIGURE 1 | Study flowchart of the 116 consecutive patients, 40 with deep neuromuscular block (NMB) are reversed by sugammadex $0.5 \mathrm{mg} / \mathrm{kg}$. Another 76 patients with moderate NMB or recovery are reversed by sugammadex $0.25 \mathrm{mg} / \mathrm{kg}$. TOF, train of four. 
TABLE 1 | Departmental anesthesia protocol for intraoperative neuromonitoring during thyroid surgery.

\begin{tabular}{|c|c|}
\hline Time courses & Remarks \\
\hline Preoperative evaluation & ASA physical status and upper airway management assessment \\
\hline Monitoring setup & Standard physical/neuromuscular/anesthesia depth monitors \\
\hline \multicolumn{2}{|l|}{ Anesthesia induction } \\
\hline Induction & Fentanyl $1 \mu \mathrm{g} / \mathrm{kg}$, lidocaine $1 \mathrm{mg} / \mathrm{kg}$, and propofol $1.5-2 \mathrm{mg} / \mathrm{kg}$ \\
\hline NMBA & Rocuronium $0.6 \mathrm{mg} / \mathrm{kg}$ \\
\hline Anesthesia maintenance & Avoid NMBA \\
\hline Inhaled anesthetic & Sevoflurane $1 \%-4 \%$ \\
\hline Intravenous anesthetic & Propofol TCl, effect-site concentration: $1 \sim 1.5 \mu \mathrm{g} / \mathrm{kg}$ \\
\hline Anesthesia depth & Entropy or BIS between 40 and 60 \\
\hline Vasopressor & Ephedrine $8-10 \mathrm{mg}$ if MAP reduction $>20 \% \mathrm{mmHg}$ \\
\hline PONV prophylaxis & Dexamethasone 5 mg, avoid morphine \\
\hline Inadequate relaxation & A bolus of fentanyl $0.5 \mu \mathrm{g} / \mathrm{kg}$ and propofol $20-30 \mathrm{mg}$ \\
\hline Neural monitoring & Low dose of sugammadex blockade \\
\hline \multirow[t]{2}{*}{ Placing TC electrodes } & Sugammadex 0.5 mg/kg if TOF count = 0 (con) \\
\hline & Sugammadex $0.25 \mathrm{mg} / \mathrm{kg}$ if TOF count $=1-4$ \\
\hline$V_{1}$ and $V_{2}$ signal & EMG amplitude correlated with TOF ratio \\
\hline \multicolumn{2}{|l|}{ Anesthesia emergency } \\
\hline \multirow[t]{2}{*}{ Extubation } & Additional sugammadex up to 2.0 mg/kg \\
\hline & Extubation when spontaneous breath with TOF ratio >0.95 \\
\hline \multirow[t]{2}{*}{ Pain control } & Parecoxib 40 mg or NSAID if not contraindicated \\
\hline & Fentanyl $0.5 \mu \mathrm{g} / \mathrm{kg}$ \\
\hline Postoperative visit & Anesthesia adverse events and satisfaction \\
\hline
\end{tabular}

ASA, American Society of Anesthesiologists; NMBA, neuromuscular blocking agent; TCl, target-controlled infusion; MAP, mean arterial pressure; BIS, bispectral index; PONV, postoperative nausea vomiting; TC, thyroid cartilage; $V_{1}$ and $V_{2}$, initial and final vagal stimulation; TOF, train-of-four mode of neuromuscular transmission monitoring; EMG, electromyography; NSAID, non-steroidal anti-inflammatory drug.

\section{Outcome Measures}

The primary outcomes of this study were the quality of the IONM and EMG amplitude of the $V_{1}$ signal. The quality of IONM was measured using the obtained $V_{1}$ amplitude. Satisfactory and weak signal was defined as a $\mathrm{V}_{1}$ amplitude of $>500 \mu \mathrm{V}$ and $<500 \mu \mathrm{V}$, respectively. The secondary outcome was the quality of surgical relaxation. The quality of relaxation was assessed by the number of free periods without any intraoperative limb movement, coughing, and swallowing events. The data of NMB, anesthesia depth, hemodynamics, postoperative adverse events, and surgical outcomes were also recorded and analyzed. All patients received preoperative and postoperative video recordings of vocal cord mobility by flexible laryngofiberoscopy. When asymmetric cord movement was found postoperatively, a comparison with the preoperative recording was performed.

\section{Statistical Analysis}

Continuous data were presented as mean \pm standard deviation (SD) values, and nominal data are presented as number (\%). The distribution of variables was tested using the KolmogorovSmirnov test. Statistical analysis of continuous variables with normal distribution between groups was compared using the unpaired t-test, while continuous variables without normal distribution were compared using the Mann-Whitney $U$ test. All statistical tests were two-tailed. Categorical nominal variables were analyzed using the chi-square or Fisher exact test. Statistical significance was set at $P<0.05$.

The sample size estimation was based on two similarly designed studies. To ensure adequate power for the study, a minimal sample size of 40 patients was used to measure the effect of $0.5 \mathrm{mg} / \mathrm{kg}$ sugammadex according to a previous study (31).
One previous study showed satisfactory nerve monitoring $\left(\mathrm{V}_{1}\right.$ $>500 \mu \mathrm{V})$ in $90 \%$ of patients when the vagus nerve was monitored after delivery of $1 \mathrm{mg} / \mathrm{kg}$ sugammadex (32). To establish a non-inferiority study in which $0.25 \mathrm{mg} / \mathrm{kg}$ sugammadex would not be less satisfactory, the case number required for the study was 38 patients per group with a $10 \%$ noninferiority margin, an alpha of 0.05 , and a beta of 0.2 .

\section{RESULTS}

A total of 116 patients ( 24 men and 92 women; aged $21-88$ years) were included in this study (Figure 1). Detailed patient characteristics are shown in Table 2. All tracheal intubations were successful after the first attempt, and no intubation-related upper airway trauma was noted. There was no difference between the two groups in terms of demographic data, physical status, disease diagnosis, vasopressor use, and surgical relaxation. The number of patients with complete surgical relaxation was significantly lower after sugammadex injection [71 (93.4\%) to $55(72.3 \%)$ in group I, $P<0.01$, vs. $39(97.5 \%)$ to $33(82.5 \%)$ in group II, $P=0.03$ ]. Overall, 193 nerves were at risk, and only one nerve had intraoperative loss of signal. This was a cancer patient who had postoperative temporary RLN palsy who recovered after 4 weeks.

Key time intervals for anesthesia, operation, and neuromonitoring did not differ significantly between the groups (Table 3). The average time from anesthesia induction (rocuronium) to sugammadex injection was $46.0( \pm 9.1) \mathrm{min}$ in group I and $43.4( \pm 9.3) \mathrm{min}$ in group II $(P=0.14)$. The average time from the surgeon's request to administer sugammadex to initial 
TABLE 2 | Patient characteristics of 116 patients receiving monitored thyroidectomy.

\begin{tabular}{|c|c|c|c|}
\hline & $\begin{array}{l}\text { Group I } \\
(n=76)\end{array}$ & $\begin{array}{l}\text { Group II } \\
(n=40)\end{array}$ & $\begin{array}{c}P \\
\text { value }\end{array}$ \\
\hline Female gender & 62 (81.5\%) & 30 (75\%) & 0.71 \\
\hline Age, mean (SD), years & $51.2(13.4)$ & $55.6(12.6)$ & 0.82 \\
\hline Weight (kg) & $60.0(11.5)$ & $57.8(9.9)$ & 0.29 \\
\hline Height (cm) & $159.8(7.7)$ & $157.7(7.3)$ & 0.14 \\
\hline BMI $\left(\mathrm{kg} / \mathrm{m}^{2}\right)$ & $23.4(3.6)$ & $23.2(3.5)$ & 0.79 \\
\hline \multicolumn{4}{|l|}{ ASA status } \\
\hline I & 2 (2.6\%) & $1(2.5 \%)$ & 0.89 \\
\hline$\|$ & $56(73.7 \%)$ & $31(77.5 \%)$ & \\
\hline III & $18(23.7 \%)$ & $8(20 \%)$ & \\
\hline Diagnosis & & & 0.75 \\
\hline Cancer & 30 (39.5\%) & $16(42.5 \%)$ & \\
\hline Benign & 46 (60.5\%) & $24(57.5 \%)$ & \\
\hline Vasopressor & 7 (9.2\%) & 5 (12.5\%) & 0.58 \\
\hline \multicolumn{4}{|l|}{ Complete relaxation* } \\
\hline Before sugammadex & 71 (93.4\%) & 39 (97.5\%) & 0.35 \\
\hline After sugammadex & 55 (72.3\%) & 33 (82.5\%) & 0.23 \\
\hline Nerve at risk (n) & 122 & 71 & \\
\hline RLN signal loss & $1(0.8 \%)$ & $0(0 \%)$ & 0.44 \\
\hline Temporary palsy & $1(0.8 \%)$ & $0(0 \%)$ & 0.44 \\
\hline Permanent palsy & $0(0 \%)$ & $0(0 \%)$ & 1.0 \\
\hline
\end{tabular}

ASA status, American Society of Anesthesiologists Physical Status classification system; BMI, body mass index (calculated as weight in kilograms divided by height in meters squared); *Without any one event of limb movement, coughing, or swallowing; " signal loss was defined as an EMG amplitude decrease of more than $50 \%$ of the baseline value.

vagal stimulation was as short as $5.4( \pm 2.1) \mathrm{min}$ in group I and 5.1 $( \pm 2.1) \mathrm{min}$ in group II $(P=0.48)$.

With respect to IONM quality, all patients showed a positive $\mathrm{V}_{1}$ signal after sugammadex. The $\mathrm{V}_{1}$ EMG amplitude was greater than $500 \mu \mathrm{V}$ in most patients. There was no significant difference between the groups (satisfactory/weak: $75 / 1$ in group I and $38 / 2$ in group II, $P=0.23$ ). The EMG amplitude and $\mathrm{NMB}$ degree were compared at $\mathrm{V}_{1}$ and $\mathrm{V}_{2}$ stimulations between the two groups. At the $V_{1}$ time point, group I demonstrated a higher EMG amplitude $[1,926( \pm 806)$ vs. $1,616( \pm 939), P=0.06]$ and a higher TOF ratio $[36( \pm 28)$ vs. 30 ( \pm 32$), P=0.29$ ] than group II, but the difference was not statistically significant (Table 3 ). Both the mean EMG amplitude and TOF ratio at the $V_{2}$ time point were comparable between the two groups (Table 3 ).

\section{DISCUSSION}

The present results revealed that both $0.5 \mathrm{mg} / \mathrm{kg}$ sugammadex for deep NMB and $0.25 \mathrm{mg} / \mathrm{kg}$ sugammadex for moderate NMB could be feasible for monitored thyroidectomy. Both groups were comparable in terms of surgical relaxation quality in terms of freedom from intraoperative limb movements, coughing, and swallowing. To the best of our knowledge, this is the first report evaluating the effects of sugammadex titrated

TABLE 3 | Time interval of procedures and quality of intraoperative neural monitoring comparison of neuromuscular blockade degree, neural monitoring recordings, and postoperative adverse events.

\begin{tabular}{|c|c|c|c|}
\hline & $\begin{array}{l}\text { Group I } \\
(n=76)\end{array}$ & $\begin{array}{l}\text { Group II } \\
(n=40)\end{array}$ & $\begin{array}{c}P \\
\text { value }\end{array}$ \\
\hline \multicolumn{4}{|l|}{ Time interval } \\
\hline Skin incision to sugammadex & $22.2(6.2)$ & $21.2(5.4)$ & 0.38 \\
\hline Sugammadex to $V_{1}$ & $5.4(2.1)$ & $5.1(2.2)$ & 0.48 \\
\hline Sugammadex to $V_{2}$ & $27.6(12.7)$ & $31.4(12.6)$ & 0.12 \\
\hline $\mathbf{V}_{1}$ amplitude & & & 0.23 \\
\hline \multicolumn{4}{|l|}{$V_{1}$ stimulation } \\
\hline EMG amplitude $(\mu \mathrm{V})$ & $1929(806)$ & 1616 (939) & 0.06 \\
\hline TOF ratio (\%) & $36(28)$ & $30(32)$ & 0.29 \\
\hline \multicolumn{4}{|l|}{$\mathbf{V}_{2}$ stimulation } \\
\hline EMG amplitude $(\mu \mathrm{V})$ & 2084 (972) & $1868(1070)$ & 0.27 \\
\hline TOF ratio (\%) & $73(24)$ & $76(19)$ & 0.53 \\
\hline
\end{tabular}

$V_{1}$, initial vagal stimulation; $V_{2}$, final vagal stimulation; EMG, electromyography; TOF, train of four. 
according to the degree of NMB for IONM during thyroid surgery.

Two fundamental elements of successful neuromonitoring are a proper recording of electrode position and NMB recovery. With respect to electrode position, in this study, we used a pair of outer thyroid cartilage electrodes to ensure stable EMG signals (34-39) instead of electrodes of an EMG endotracheal tube, which was susceptible to rotational or depth changes $(18,38,40)$. Regarding NMB management, several feasible regimens have been proposed to facilitate tracheal intubation and functional IONM (8). Sugammadex has gained increasing popularity in IONM during thyroid surgery. The reported dose of sugammadex that was effective for IONM ranged from 0.5 to $2.0 \mathrm{mg} / \mathrm{kg}(28,29,31,32,41,42)$. There is a wide variation between patients in the spontaneous recovery time from rocuronium-induced $\mathrm{NMB}$ group. Hence, precise and timely titration of sugammadex dose according to different NMB doses could be more effective and practical during IONM.

Sugammadex at $0.5 \mathrm{mg} / \mathrm{kg}$ was reported to achieve a high EMG amplitude of $1,214( \pm 623) \mu \mathrm{V}$ at $\mathrm{V}_{1}$ stimulation during thyroidectomy (31). Complete and early reversal of NMB was very effective for IONM, providing surgical relaxation. In clinical observations, vigorous movements might occur immediately after a dose of 1 or $2 \mathrm{mg} / \mathrm{kg}$ sugammadex. Chai et al. (32) also demonstrated that sugammadex at $2 \mathrm{mg} / \mathrm{kg}$ was associated with more bucking than $1 \mathrm{mg} / \mathrm{kg}$ (35\% vs. 14\%) during thyroid surgery with IONM. Since different sugammadex doses provided comparable high-quality EMG signals, this modified NMB protocol attempted to explore minimal sugammadex doses that could allow high-quality IONM signals. In a previous report, sugammadex at $0.5 \mathrm{mg} / \mathrm{kg}$ was effective for deep $\mathrm{NMB}$, and sugammadex dose at $0.25 \mathrm{mg} / \mathrm{kg}$ provided moderate $\mathrm{NMB}$ or recovery. A reduction in sugammadex dose for a lower degree of NMB was found to be effective for high-quality IONM $\left(\mathrm{V}_{1}\right.$ amplitude $\left.>500 \mu \mathrm{V}\right)$ in $98.7 \%(75 / 76)$ of patients. Although there was one patient with a $\mathrm{V}_{1}$ signal of $<500 \mu \mathrm{V}(445$ $\mu \mathrm{V}$ ), typical EMG waveforms can be easily observed, and IONM was successfully performed without difficulty.

In addition to the dose of sugammadex, the timing of administration plays a key role in NMB management. Sugammadex has been developed to selectively bind to aminosteroidal NMBAs (i.e., rocuronium) selectively $(43,44)$. Sugammadex provides rapid and effective reversal of rocuroniuminduced NMB not only for residual NMB after extubation but also for high-quality IONM signals during surgery. When considering the rapid time of onset, the interval between $V_{1}$ and sugammadex in this protocol was modified to be as short as possible. This study employed a team approach during IONM, wherein the anesthesiologist administered sugammadex per the surgeon's request after exposing the anterior surface of the thyroid gland and preparing for initial $\left(\mathrm{V}_{1}\right)$ vagal stimulation. The overall interval from sugammadex administration to $\mathrm{V}_{1}$ stimulation was $5.32( \pm 2.2) \mathrm{min}$.

There is a lack of consensus regarding sugammadex timing before vagal stimulation; several time points reported for administration were as follows: immediately after tracheal tube fixation, at skin incision, at $10 \mathrm{~min}$ after skin excision, and at exposure and identification of the vagus nerve. The interval from sugammadex to V1 stimulation was between 3 and $32 \min (9,12$, $13,22)$. In a selective protocol, sugammadex was used after $V_{1}$ stimulation when the EMG amplitude was absent or below 100 $\mu \mathrm{V}$. However, a low initial $\mathrm{V}_{1}$ amplitude limits the application of the IONM troubleshooting algorithm in case of signal loss (10). Sugammadex timing may affect the reversal of NMB. In this study, the mean TOF ratio was $30 \%$ at $\mathrm{V}_{1}$ stimulation after sugammadex $0.5 \mathrm{mg} / \mathrm{kg}$ was administered before $\mathrm{V}_{1}$ stimulation. In our previous report, the TOF ratio was $59 \%$ after sugammadex $0.5 \mathrm{mg} / \mathrm{kg}$ was administered $10 \mathrm{~min}$ after starting the operation (12). Both regimens were able to present comparable highquality IONM signals during thyroid surgery.

There are several advantages to this surgeon-centered NMB degree titrated sugammadex protocol for monitored thyroidectomy. First, sugammadex titration based on NMB degree provided two effective rocuronium doses $(0.6 \mathrm{mg} / \mathrm{kg})$ to provide an excellent condition for tracheal intubation in most patients (9, 12). Second, sugammadex administration approximately $5 \mathrm{~min}$ before $\mathrm{V}_{1}$ stimulation ensured an adequate onset time to reverse NMB. The protocol maximizes the sugammadex-free interval to avoid bucking or movement resulting from a lower degree of NMB (13). Finally, regarding IONM quality, a high EMG amplitude was noted in 97.4\% (113/ 116 ) of patients. An initial high-quality $V_{1}$ signal is crucial to the signal loss algorithm because the initial EMG amplitude is a standard reference to be compared $(1,5,45,46)$ and to detect imminent nerve stress or injury $(11,12,47-50)$.

In addition to the application of sugammadex, several alternative methods could be used for IONM during thyroid surgery. Since sugammadex is not available in all institutes because of its high cost, dose titration of neuromuscular blocking agents might also enable tracheal intubation and surgical relaxation. In a review of NMB management for IONM without using sugammadex, regimens including relaxant-free, succinylcholine, and low to standard dose of rocuronium $(0.3 \sim 0.6 \mathrm{mg} / \mathrm{kg})$ were all feasible with some clinical limitations $(24,27)$.

This study had several limitations. First, the study design lacked patient randomization and blinding regarding the administered sugammadex dose because of grouping based on the degree of NMB. Patients in both groups showed comparable demography, disease, and procedure profiles. Second, there might be a selection bias due to the high cost of sugammadex. This was a self-payment regimen; thus, patients who could afford sugammadex and had a high socioeconomic status were included. Third, this protocol was limited by the NMB monitoring equipment in addition to sugammadex. The NMB degree was measured by the TOF ratio generally via acceleromyography or kinemyography devices in Taiwan. However, both devices and sugammadex are not easily available for every institute. Finally, caution should be exercised when interpreting the outcomes in this trial. The outcomes were based on the close cooperation between the surgery and the anesthesia team with much clinical and laboratory experience in thyroid surgery with IONM. The IONM outcomes may be influenced by team members in various clinical backgrounds. 


\section{CONCLUSIONS}

Before vagal stimulation, this surgeon-centered sugammadex protocol according to NMB degree allowed high IONM quality and adequate surgical relaxation. Both sugammadex $0.5 \mathrm{mg} / \mathrm{kg}$ for deep NMB and $0.25 \mathrm{mg} / \mathrm{kg}$ for moderate NMB or less showed comparable high EMG amplitude at $\mathrm{V}_{1}$ stimulation. Sugammadex titration for reversal of NMB prevented undetectable or markedly low EMG amplitudes whenever nerve stimulation was required during thyroidectomy. Moreover, the flexibility of sugammadex administration timing based on the surgical procedure was also feasible for IONM during thyroid surgery. These initial positive results warrant further randomized controlled trials.

\section{DATA AVAILABILITY STATEMENT}

The raw data supporting the conclusions of this article will be made available by the authors without undue reservation.

\section{ETHICS STATEMENT}

The studies involving human participants were reviewed and approved by the institutional review board of Kaohsiung Medical University Hospital, Kaohsiung Taiwan. Written informed consent for participation was not required for this study in accordance with the national legislation and institutional requirements.

\section{REFERENCES}

1. Randolph GW, Dralle H, Abdullah H, Barczynski M, Bellantone R, Brauckhoff $M$, et al. Electrophysiologic Recurrent Laryngeal Nerve Monitoring During Thyroid and Parathyroid Surgery: International Standards Guideline Statement. Laryngoscope (2011) 121(Suppl 1):S1-16. doi: 10.1002/lary.21119

2. Sanabria A, Kowalski LP, Nixon I, Angelos P, Shaha A, Owen RP, et al. Methodological Quality of Systematic Reviews of Intraoperative Neuromonitoring in Thyroidectomy: A Systematic Review. JAMA Otolaryngol Head Neck Surg (2019) 145(6):563-73. doi: 10.1001/jamaoto. 2019.0092

3. Feng AL, Puram SV, Singer MC, Modi R, Kamani D, Randolph GW. Increased Prevalence of Neural Monitoring During Thyroidectomy: Global Surgical Survey. Laryngoscope (2020) 130(4):1097-104. doi: 10.1002/lary.28210

4. Genther DJ, Kandil EH, Noureldine SI, Tufano RP. Correlation of Final Evoked Potential Amplitudes on Intraoperative Electromyography of the Recurrent Laryngeal Nerve With Immediate Postoperative Vocal Fold Function After Thyroid and Parathyroid Surgery. JAMA Otolaryngol Head Neck Surg (2014) 140(2):124-8. doi: 10.1001/jamaoto.2013.6139

5. Wu CW, Randolph GW, Barczyński M, Schneider R, Chiang FY, Huang TY, et al. Training Courses in Laryngeal Nerve Monitoring in Thyroid and Parathyroid Surgery- The INMSG Consensus Statement. Front Endocrinol (Lausanne) (2021) 12:705346:705346. doi: 10.3389/fendo.2021.705346

6. Fundakowski CE, Hales NW, Agrawal N, Barczyński M, Camacho PM, Hartl $\mathrm{DM}$, et al. Surgical Management of the Recurrent Laryngeal Nerve in Thyroidectomy: American Head and Neck Society Consensus Statement. Head Neck (2018) 40(4):663-75. doi: 10.1002/hed.24928

7. Vasileiadis I, Karatzas T, Charitoudis G, Karakostas E, Tseleni-Balafouta S, Kouraklis G. Association of Intraoperative Neuromonitoring With Reduced

\section{AUTHOR CONTRIBUTIONS}

C-WW had full access to all the data in the study and takes responsibility for the integrity of the data and the accuracy of the data analysis. Y-WK and C-WW contributed equally to this work. Concept and design: I-CL and C-WW. Acquisition, analysis, or interpretation of data: I-CL, C-DH, P-YC, GD, YJC, C-WW. Drafting of the article: I-CL, F-YC, Y-WK, C-WW. Critical revision of the article and final approval: All authors. Statistical analysis: I-CL, F-YC, Y-CL, C-WW. Obtained funding: I-CL, T-YH, C-WW. Administrative, technical, or material support: S-HW, TYH, Y-CL, H-YK, and F-YC. Supervision: Y-WK and C-WW.

\section{FUNDING}

This study was supported by grants from the Kaohsiung Municipal Siaogang Hospital (H-109-001, Kmhk-110-08) and the Ministry of Science and Technology, Taiwan (MOST 1092314-B-037-059).

\section{ACKNOWLEDGMENTS}

The authors express gratitude to Hsiu-Ya Chen (nurse anesthetist, Department of Anesthesiology, KMUH, KMU), Hui-Chun Chen (clinical nurse specialist, Department of Nursing, KMUH, KMU), and Pao-Chu Hun (veterinarian, Laboratory Animal Center, KMU) for their excellent technical assistance.

Recurrent Laryngeal Nerve Injury in Patients Undergoing Total Thyroidectomy. JAMA Otolaryngol Head Neck Surg (2016) 142(10):9941001. doi: 10.1001/jamaoto.2016.1954

8. Patel KN, Yip L, Lubitz CC, Grubbs EG, Miller BS, Shen W, et al. The American Association of Endocrine Surgeons Guidelines for the Definitive Surgical Management of Thyroid Disease in Adults. Ann Surg (2020) 271(3): e21-93. doi: 10.1097/sla.0000000000003580

9. Al-Qurayshi Z, Randolph GW, Alshehri M, Kandil E. Analysis of Variations in the Use of Intraoperative Nerve Monitoring in Thyroid Surgery. JAMA Otolaryngol Head Neck Surg (2016) 142(6):584-9. doi: 10.1001/jamaoto. 2016.0412

10. Abdelhamid A, Aspinall S. Intraoperative Nerve Monitoring in Thyroid Surgery: Analysis of United Kingdom Registry of Endocrine and Thyroid Surgery Database. Br J Surg (2021) 108(2):182-7. doi: 10.1093/bjs/znaa081

11. Schneider R, Randolph GW, Dionigi G, Wu CW, Marcin Barczynski M, Chiang FU, et al. International Neural Monitoring Study Group Guideline 2018 Part I: Staging Bilateral Thyroid Surgery With Monitoring Loss of Signal. Laryngoscope (2018) 128(Suppl 3):S1-17. doi: 10.1002/lary.27359

12. Wu CW, Dionigi G, Barczynski M, Chiang FY, Dralle H, Schneider R, et al. International Neuromonitoring Study Group Guidelines 2018: Part II: Optimal Recurrent Laryngeal Nerve Management for Invasive Thyroid Cancer-Incorporation of Surgical, Laryngeal, and Neural Electrophysiologic Data. Laryngoscope (2018) 128(Suppl 3):S18-27. doi: 10.1002/lary.27360

13. Lu IC, Chu KS, Tsai CJ, Wu CW, Kuo WR, Chen HY, et al. Optimal Depth of NIM EMG Endotracheal Tube for Intraoperative Neuromonitoring of the Recurrent Laryngeal Nerve During Thyroidectomy. World J Surg (2008) 32 (9):1935-9. doi: 10.1007/s00268-008-9549-1

14. Berkow L, Dackiw AP, Tufano RP. Use of the GlideScope for Placement of a Recurrent Laryngeal Nerve Monitoring Endotracheal Tube. J Clin Anesth (2011) 23(1):81-3. doi: 10.1016/j.jclinane.2009.12.013 
15. Tsai CJ, Tseng KY, Wang FY, Lu IC, Wang HM, Wu CW, et al. Electromyographic Endotracheal Tube Placement During Thyroid Surgery in Neuromonitoring of Recurrent Laryngeal Nerve. Kaohsiung J Med Sci (2011) 27(3):96-101. doi: 10.1016/j.kjms.2010.08.002

16. Kanotra SP, Kuriloff DB, Lesser J, Rest-Flarer F. GlideScope-Assisted Nerve Integrity Monitoring Tube Placement for Intra-Operative Recurrent Laryngeal Nerve Monitoring. J Laryngol Otol (2012) 126(12):1271-3. doi: $10.1017 /$ S0022215112002460

17. Cherng CH, Huang YH, Shih ML. Middle Fixation of Electromyographic Endotracheal Tube for Intraoperative Recurrent Laryngeal Nerve Monitoring. J Clin Anesth (2014) 26(3):252-3. doi: 10.1016/j.jclinane.2014.01.009

18. Barber SR, Liddy W, Kyriazidis N, Cinquepalmi M, Brian M Lin BM, Modi R, et al. Changes in Electromyographic Amplitudes But Not Latencies Occur With Endotracheal Tube Malpositioning During Intraoperative Monitoring for Thyroid Surgery: Implications for Guidelines. Laryngoscope (2017) 127 (9):2182-8. doi: 10.1002/lary.26392

19. Huang JM, Hsu CD, Wu SH, Kuo YW, Huang TY, Wu CW, et al. Optimization of Electromyographic Endotracheal Tube Electrode Position by UEScope for Monitored Thyroidectomy. Laryngoscope Investig Otolaryngol (2021) 6(5):1214-19. doi: 10.1002/lio2.635

20. Marusch F, Hussock J, Haring G, Hachenberg T, Gastinger I. Influence of Muscle Relaxation on Neuromonitoring of the Recurrent Laryngeal Nerve During Thyroid Surgery. Br J Anaesth (2005) 94(5):596-600. doi: 10.1093/bja/ aeil10

21. Shi Y, Hou V, Tucker A, Cohen J. Changes of Extremity and Laryngeal Muscle Electromyographic Amplitudes After Intravenous Administration of Vecuronium. Laryngoscope (2008) 118(12):2156-60. doi: 10.1097/ MLG.0b013e318184574e

22. Chu KS, Wu SH, Lu IC, Tsai CJ, Wu CW, Kuo WR, et al. Feasibility of Intraoperative Neuromonitoring During Thyroid Surgery After Administration of Nondepolarizing Neuromuscular Blocking Agents. World J Surg (2009) 33(7):1408-13. doi: 10.1007/s00268-009-0049-8

23. Chu KS, Tsai CJ, Lu IC, Tseng KY, Chau SW, Wu CW, et al. Influence of Nondepolarizing Muscle Relaxants on Intraoperative Neuromonitoring During Thyroid Surgery. J Otolaryngol Head Neck Surg (2010) 39(4):397-402.

24. Lu IC, Tsai CJ, Wu CW, Cheng KI, Wang FY, Tseng KY, et al. A Comparative Study Between 1 and 2 Effective Doses of Rocuronium for Intraoperative Neuromonitoring During Thyroid Surgery. Surgery (2011) 149(4):543-8. doi: 10.1016/j.surg.2010.11.006

25. Brunaud L, Fuchs-Buder T. In Reference to Reversal of Rocuronium-Induced Neuromuscular Blockade by Sugammadex Allows for Optimization of Neural Monitoring of the Recurrent Laryngeal Nerve. Laryngoscope (2017) 127(1): E50. doi: 10.1002/lary.25990

26. Lu IC, Lin IH, Wu CW, Chen HY, Lin YC, Chiang FY, et al. Preoperative, Intraoperative and Postoperative Anesthetic Prospective for Thyroid Surgery: What's New. Gland Surg (2017) 6(5):469-75. doi: 10.21037/gs.2017.05.02

27. Lu IC, Wu SH, Wu CW. Neuromuscular Blockade Management for Intraoperative Neural Monitoring. Kaohsiung J Med Sci (2020) 36(4):230-5. doi: $10.1002 / \mathrm{kjm} 2.12153$

28. Lu IC, Wu CW, Chang PY, Chen HY, Tseng KY, Randolph GW, et al. Reversal of Rocuronium-Induced Neuromuscular Blockade by Sugammadex Allows for Optimization of Neural Monitoring of the Recurrent Laryngeal Nerve. Laryngoscope (2016) 126(4):1014-9. doi: 10.1002/lary.25577

29. Empis de Vendin O, Schmartz D, Brunaud L, Fuchs-Buder T. Recurrent Laryngeal Nerve Monitoring and Rocuronium: A Selective Sugammadex Reversal Protocol. World J Surg (2017) 41(9):2298-303. doi: 10.1007/ s00268-017-4004-9

30. Wu CW, Huang TY, Chen HC, Chen HY, Tsai TY, Chang PY, et al. IntraOperative Neural Monitoring of Thyroid Surgery in a Porcine Model. J Vis Exp (2019) 144:e57919. doi: 10.3791/57919

31. Lu IC, Wu SH, Chang PY, Ho PY, Huang TY, Lin YC, et al. Precision Neuromuscular Block Management for Neural Monitoring During Thyroid Surgery. J Invest Surg (2020) 34(12):1389-96. doi: 10.1080/08941939. 2020.1805055

32. Chai YJ, Lee JM, Won D, Jiwon Lee J, Hwang JY, Kim TK, et al. Comparison of Sugammadex Dose for Intraoperative Neuromonitoring in Thyroid Surgery: A Randomized Controlled Trial. Laryngoscope (2021) 131 (9):2154-9. doi: 10.1002/lary.29515
33. Fuchs-Buder T, Meistelman C, Raft J. Sugammadex: Clinical Development and Practical Use. Korean J Anesthesiol (2013) 65(6):495-500. doi: 10.4097/ kjae.2013.65.6.495

34. Chiang FY, Wu CW, Chang PY, Wu SH, Chen HY, Lin YC, et al. TransThyroid Cartilage Recording for Neural Monitoring of the Recurrent Laryngeal Nerve in Thyroid Surgery. Laryngoscope (2020) 130(4):E280-3. doi: 10.1002/lary.28049

35. Türk Y, Kıvratma G, Özdemir M, İçöz G, Makay Ö. The Use of Thyroid Cartilage Needle Electrodes in Intraoperative Neuromonitoring During Thyroidectomy: A Case-Control Study. Head Neck (2021) 43(11):3287-93. doi: 10.1002/hed.26810

36. Jung SM, Tae K, Song CM, Lee SH, Jeong JH, Ji YB. Efficacy of Transcartilaginous Electrodes for Intraoperative Neural Monitoring During Thyroid Surgery. Clin Exp Otorhinolaryngol (2020) 13(4):422-8. doi: 10.21053/ceo.2019.01529

37. Liddy W, Lawson BR, Barber SR, Kamani D, Shama M, Soylu S, et al. Anterior Laryngeal Electrodes for Recurrent Laryngeal Nerve Monitoring During Thyroid and Parathyroid Surgery: New Expanded Options for Neural Monitoring. Laryngoscope (2018) 128(12):2910-5. doi: 10.1002/ lary. 27362

38. Chiang FY, Lu IC, Chang PY, Dionigi G, Randolph GW, Sun H, et al. Comparison of EMG Signals Recorded by Surface Electrodes on Endotracheal Tube and Thyroid Cartilage During Monitored Thyroidectomy. Kaohsiung J Med Sci (2017) 33(10):503-9. doi: 10.1016/ j.kjms.2017.06.014

39. Huang TY, Kim HY, Dionigi G, Lu IC, Chang PY, Chiang FY, et al. Laryngeal Neural Monitoring During Pediatric Thyroid Cancer Surgery-Is Transcartilage Recording a Preferable Method? Cancers (2021) 13(16):4051. doi: $10.3390 /$ cancers 13164051

40. Kim HY, Tufano RP, Randolph G, Marcin Barczyński M, Wu CW, Chiang FY, et al. Impact of Positional Changes in Neural Monitoring Endotracheal Tube on Amplitude and Latency of Electromyographic Response in Monitored Thyroid Surgery: Results From the Porcine Experiment. Head Neck (2016) 38 (Suppl 1):E1004-8. doi: 10.1002/hed.24145

41. Donmez T, Erdem VM, Sunamak O, Ozcevik H. Thyroid Surgery, Ionm and Sugammadex Sodium Relationships: Benefits in Sugammadex Sodium Use for Ionm. Acta Endocrinol (Buchar) (2019) 15(4):454-9. doi: 10.4183/ aeb. 2019.454

42. Gunes ME, Dural AC, Akarsu C, Guzey D, Sahbaz NA, Tulubas EK, et al. Effect of Intraoperative Neuromonitoring on Efficacy and Safety Using Sugammadex in Thyroid Surgery: Randomized Clinical Trial. Ann Surg Treat Res (2019) 97(6):282-90. doi: 10.4174/astr.2019.97.6.282

43. Kovac AL. Sugammadex: The First Selective Binding Reversal Agent for Neuromuscular Block. J Clin Anesth (2009) 21(6):444-53. doi: 10.1016/ j.jclinane.2009.05.002

44. Yang LP, Keam SJ. Sugammadex: A Review of its Use in Anaesthetic Practice. Drugs (2009) 69(7):919-42. doi: 10.2165/00003495-200969070-00008

45. Chiang FY, Lu IC, Kuo WR, Lee KW, Chang NC, Wu CW. The Mechanism of Recurrent Laryngeal Nerve Injury During Thyroid Surgery-the Application of Intraoperative Neuromonitoring. Surgery (2008) 143(6):743-9. doi: 10.1016/ j.surg.2008.02.006

46. Chiang FY, Lee KW, Chen HC, Chen HY, Lu IC, Kuo WR, et al Standardization of Intraoperative Neuromonitoring of Recurrent Laryngeal Nerve in Thyroid Operation. World J Surg (2010) 34(2):223-9. doi: 10.1007/ s00268-009-0316-8

47. Wu CW, Wang MH, Chen CC, Chen HC, Chen HY, Yu JY, et al. Loss of Signal in Recurrent Nerve Neuromonitoring: Causes and Management. Gland Surg (2015) 4(1):19-26. doi: 10.3978/j.issn.2227684X.2014.12.03

48. Wu CW, Hao M, Tian M, Dionigi G, Tufano RP, Kim HY, et al. Recurrent Laryngeal Nerve Injury With Incomplete Loss of Electromyography Signal During Monitored Thyroidectomy-Evaluation and Outcome. Langenbecks Arch Surg (2017) 402(4):691-9. doi: 10.1007/s00423016-1381-8

49. Wu CW, Dionigi G, Sun H, Liu X, Kim HY, Hsiao PJ, et al. Intraoperative Neuromonitoring for the Early Detection and Prevention of RLN Traction Injury in Thyroid Surgery: A Porcine Model. Surgery (2014) 155(2):329-39. doi: 10.1016/j.surg.2013.08.015 
50. Schneider R, Randolph GW, Sekulla C, Phelan E, Thanh PN, Bucher M, et al. Continuous Intraoperative Vagus Nerve Stimulation for Identification of Imminent Recurrent Laryngeal Nerve Injury. Head Neck (2013) 35 (11):1591-8. doi: 10.1002/hed.23187

Conflict of Interest: The authors declare that the research was conducted in the absence of any commercial or financial relationships that could be construed as a potential conflict of interest.

Publisher's Note: All claims expressed in this article are solely those of the authors and do not necessarily represent those of their affiliated organizations, or those of the publisher, the editors and the reviewers. Any product that may be evaluated in this article, or claim that may be made by its manufacturer, is not guaranteed or endorsed by the publisher.

Copyright $\odot 2022$ Lu, Hsu, Chang, Wu, Huang, Lin, Ko, Dionigi, Chai, Chiang, Kuo and $W u$. This is an open-access article distributed under the terms of the Creative Commons Attribution License (CC BY). The use, distribution or reproduction in other forums is permitted, provided the original author(s) and the copyright owner(s) are credited and that the original publication in this journal is cited, in accordance with accepted academic practice. No use, distribution or reproduction is permitted which does not comply with these terms. 